\title{
Follow-up Visits and Changes in Pain Scores Reported by Oncology Outpatients After Initial Presentation With Severe Pain
}

\author{
Brett Hill ${ }^{1}$, Dwight Moulin ${ }^{2}$, Michael Sanatani ${ }^{1}$ \\ 1. Medical Oncology, Schulich School of Medicine \& Dentistry, London Regional Cancer Program, \\ Western University, London, Ontario, CA 2. Neuro-oncology, Schulich School of Medicine \& Dentistry, \\ London Regional Cancer Program, Western University, London, Ontario, CA
}

$\square$ Corresponding author: Michael Sanatani, michael.sanatani@lhsc.on.ca Disclosures can be found in Additional Information at the end of the article

\section{Abstract}

\section{Background}

In addition to tumour treatment, the management of symptoms such as pain is an important component of cancer care. Pain management is a complex field and prior studies have highlighted many different clinical care responses to a cancer patient presenting with severe pain. We explored follow-up and how pain screening scores changed over time, among a cohort of cancer outpatients, and how follow-up was scheduled after the initial visit.

\section{Methods}

The care provided to 96 patients seen at the London Regional Cancer Program was reviewed for the 12-week period following presentation with severe pain $>7 / 10$. Follow-up ESAS (Edmonton Symptom Assessment System) scores, visits, and compliance were documented.

\section{Results}

Follow-up ESAS data was available for 41/96 patients. Mean ESAS pain decreased from 8.4/10 to 3.6/10 among those patients with follow-up; however, for 55/96 patients, no follow-up ESAS score was available (deceased $n=3$, no follow-up visit $n=41$, no pain score reported, $n=11$ ).

\section{Conclusions}

Despite a very high proportion of documented active pain management plans in the case of cancer patients presenting with severe pain, very little follow-up directed specifically at pain management was performed. Cancer treatment appears to be the primary determinant of oncology follow-up timing at our centre.

Received 09/07/2016 Review began 09/30/2016 Review ended 01/03/2017 Published 01/09/2017

(c) Copyright 2017

Hill et al. This is an open access article distributed under the terms of the Creative Commons Attribution License CC-BY 3.0., which permits unrestricted use, distribution, and reproduction in any medium, provided the original author and source are credited.
Categories: Pain Management, Oncology, Quality Improvement

Keywords: cancer pain, symptom management, follow-up

\section{Introduction}

Pain is one of the most prevalent symptoms in patients with metastatic cancer and has wideranging deleterious effects on activity, mood, and sleep, to name a few [1]. Quality of life is compromised when pain control is poor [1]. However, pain is commonly mismanaged and 
inadequately treated in a clinical setting [2-6]. Yennurajalingham et al. found that $50 \%$ of patients surveyed, who were experiencing moderate to severe pain, reported no pain relief following analgesia, and $32 \%$ of patients with moderate pain indicated an increase in pain. These findings have been replicated throughout the literature [2-6], indicating that work needs to be done to better understand the management of cancer pain.

Addressing some of the concerns surrounding pain management has been difficult, and the various components of the pain management process need to be defined and improved [7-9]. Assessment of pain is the first step of effective pain management. At the London Regional Cancer Program (LRCP) in Ontario, Canada, all patient visits include electronic completion of the Edmonton Symptom Assessment System (ESAS) which includes a pain scale (zero to 10). The completed form can be seen on the patient's electronic record by the nurse and physician prior to the clinic visit [10-11]. ESAS is a standardized symptom screening tool used to assess common symptoms in cancer patients: pain, tiredness, nausea, depression, anxiety, drowsiness, appetite, well-being, and shortness of breath, and a blank space for the patient to fill in any additional problem [10]. Zero represents absence of the symptom, while a score of ten represents the worst severity possible. A pain score of greater than or equal to seven is considered to be severe [10]. A decrease in two points or greater has been suggested as being clinically significant [12], while inadequate pain management is defined as no clinically significant change or an increase in pain score over time.

In a previous study at the LRCP by Sanatani et al., a high proportion (83\%) of patients who reported severe pain (ESAS $>7$ ), received an active intervention targeted at managing that pain [11]. Further study is needed to determine the subsequent effectiveness of various care approaches in improving the pain level that is initially reported. The next step is to investigate the changes, if any, over time after the initial visit where a high pain score was reported by the patient. This current study examines the timeline of the ESAS pain subscale changes over three months following the initial visit, to assess ongoing attention to pain management and any correlation with other ESAS subscales.

Informed consent was obtained from patients, and IRB approval was provided by Western University Health Sciences Research Ethics Board (approval \#106326).

\section{Materials And Methods Purpose and study endpoints}

The purpose of the present study was to determine the mean changes in ESAS pain score from baseline, occurring at follow-up visits after the implementation of various pain management regimens in patients presenting with severe pain (7-10/10). The follow-up pain scores were recorded for the visits as listed in Table 1. Associated data collected from the charts included information on whether the previously documented management plans actually were implemented, baseline demographics, and the other ESAS subscales (all rated 1-10): nausea, well-being, depression, anxiety, dyspnea, drowsiness, fatigue, and anorexia. In addition, the number of patients for whom follow-up ESAS scores were available was determined in order to assess follow-up appointment scheduling. 


\section{Cureus}

\section{Baseline}

First return visit with ESAS data available, 14 weeks after baseline visit

First return visit with ESAS data available, 4-8 weeks after baseline visit

First return visit with ESAS data available, 8-12 weeks after baseline visit

Latest return with ESAS data available, before 12 weeks from baseline

\section{TABLE 1: Pain score assessment times}

\section{Eligibility and patient selection}

A retrospective chart review was done (both electronic and paper contents) on the patients previously selected and reported on by Sanatani et al [11]. The patient medical record (both electronic and paper contents) was reviewed for these sequential unique patient visits, starting December 15, 2011, at solid tumour medical, surgical, and radiation oncology clinics at the LRCP, with documented patient pain levels $>7 / 10$ at the electronic kiosk prior to seeing the nurse and physician. Patient visit identification was done by a search of the electronic hospital records, starting with the first visit registered at our centre at 8:00 a.m. on December 15, 2011, and searching forward, screening first by severe ESAS pain level, and then by inclusion and exclusion criteria. Patients attending only the palliative care or pain management clinics were excluded from the current study because, by definition, patients attending these clinics had been specifically referred for review of an active pain management plan. Follow-up visits for administration of chemotherapy or radiation without physician involvement, urgent visits to the ambulatory care bay for dehydration, transfusions, electrolyte imbalances, or other acute issues were excluded.

\section{Results}

Ninety-six charts were found eligible for this follow-up study and were reviewed (both electronic record and paper chart including nursing documentation). Baseline characteristics are indicated in Table 2. Out of these patients, 41 had at least one follow-up ESAS score documented within 12 weeks of the baseline visit. Forty-four patients had no follow-up visit within 12 weeks of the baseline visit, and the reasons are outlined in Table 3.

\section{Recorded Data}

Age (mean, range)

$63,26-95$

Gender (M/F) $38 / 58$ 


\section{Cureus}

Primary tumour site $(\mathrm{n})$

\begin{tabular}{ll} 
Unknown & 12 \\
Breast & 20 \\
Colorectal & 10 \\
Prostate & 8 \\
Renal & 4 \\
Bladder & 2 \\
Uterine & 2 \\
Cervix & 1 \\
Pancreatic & 1 \\
Head and Neck & 10 \\
Neuroendocrine & 13 \\
Peritoneal & 2 \\
Skin & 2 \\
Gallbladder & 2 \\
Unknown Primary & 2 \\
Brain & 38 \\
Lung & 33 \\
Medical Oncology & 5 \\
Radiation Oncology & 15 \\
Surgical Oncology & 51 \\
Curative: 45 / Non-curative: 51 & 2 \\
\hline
\end{tabular}

Treatment intent $(\mathrm{n})$

TABLE 2: Baseline characteristics 


\section{Cureus}

Deceased

No follow-up booked at LRCP within 12 weeks

Did not attend booked follow-up

\section{TABLE 3: Reasons for absence of follow-up ESAS scores $(n=55)$}

\section{ESAS Pain Scores}

The changes in ESAS pain scores for those patient visits captured within the 12-week follow-up window are indicated in Table 4 and Figure 1. Over time, the mean ESAS pain score from visits in the three follow-up periods (1-4 weeks, 5-8 weeks, and 9-12 weeks after initial visit) decreased (Figure 1). However, the numbers of patients with follow-up visit ESAS scores declined, and there was a correlation between the last ESAS score recorded and the length of interval between the baseline and the last ESAS score $(r=0.379, \mathrm{p}=0.015)$. Situations where the initially planned pain management was not carried out are summarized in Table 5.

\begin{tabular}{|c|c|c|c|c|c|}
\hline & Baseline & $\begin{array}{l}\text { Follow-up } \\
\text { between } \\
\text { weeks 1-4 }\end{array}$ & $\begin{array}{l}\text { Follow-up } \\
\text { between } \\
\text { weeks 5-8 }\end{array}$ & $\begin{array}{l}\text { Follow-up } \\
\text { between weeks 9- } \\
12\end{array}$ & $\begin{array}{l}\text { Longest follow-up } \\
\text { visit available }\end{array}$ \\
\hline $\mathrm{n}$ & 96 & 23 & 16 & 16 & 41 \\
\hline Mean ESAS (SD) & $\begin{array}{l}8.43 \\
(1.08)\end{array}$ & $5.61(2.98)$ & $4.25(3.34)$ & $3.56(2.68)$ & 4.78 (3.29) \\
\hline $\begin{array}{l}\text { Mean ESAS change } \\
\text { from baseline (SD) }\end{array}$ & $\mathrm{n} / \mathrm{a}$ & $2.96(2.82)$ & $4.25(3.61)$ & $4.69(2.70)$ & 3.59 (3.24) \\
\hline $\begin{array}{l}\text { Median time from } \\
\text { baseline visit (days) }\end{array}$ & $\mathrm{n} / \mathrm{a}$ & 13.0 & 37.5 & 69.0 & 42.0 \\
\hline
\end{tabular}

\section{TABLE 4: Pain scores over time}




\section{Cureus}

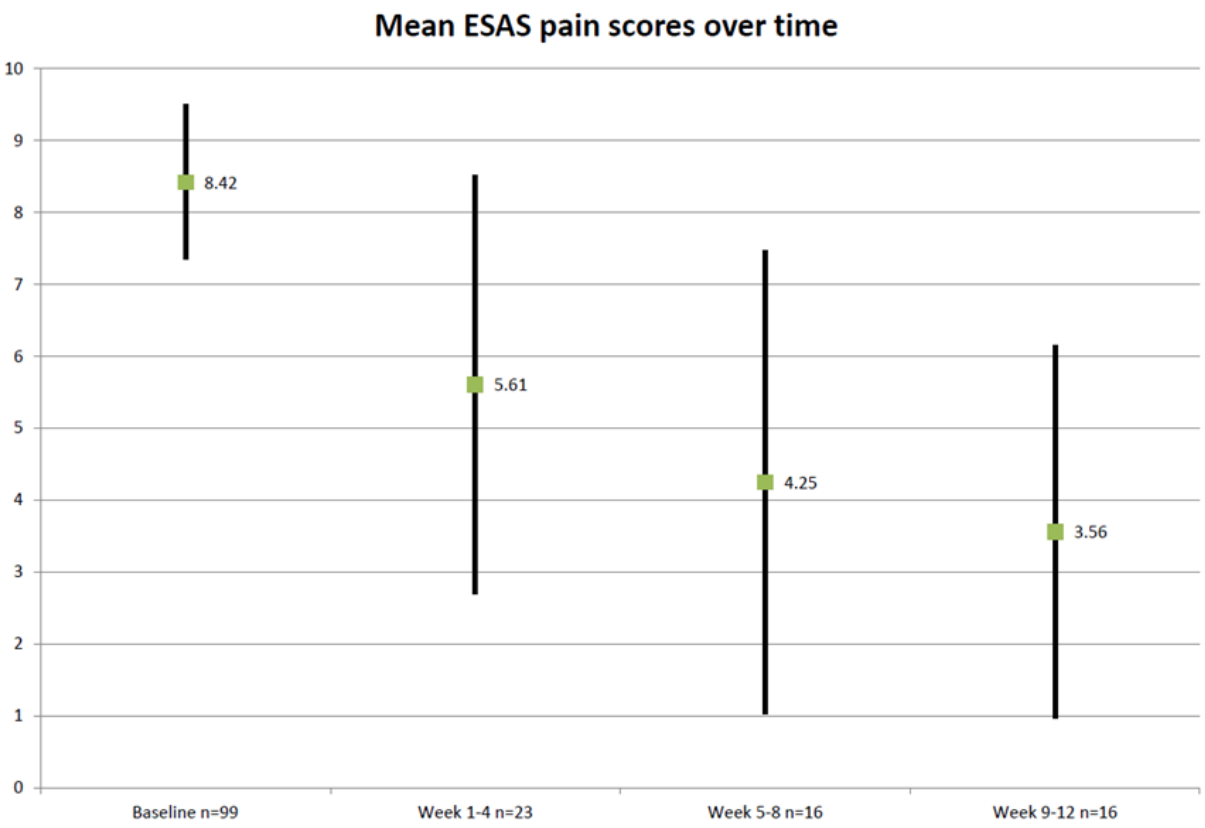

FIGURE 1: Changes in ESAS pain scores over time

Discrepancy

Patient did not take or stopped prescribed medication - lack of perceived effect

Patient did not take or stopped prescribed medication - adverse effect, ran out, or took other medication instead 4

Unforeseen acute event (fall, hospital admission)

Patient declined recommended treatment or consultation

Treatment plan changed by physician

Pain resolved

TABLE 5: Discrepancies between original pain management plan and actual care $(n=16)$

Associations between patient characteristics and the change in ESAS pain score from baseline to longest follow-up were examined. No significant predictive factors were found.

\section{Discussion}

In this follow-up study, 96 patient charts were reviewed to assess the changes in pain scores reported by cancer patients after a baseline visit where they had indicated their pain was severe 
$(>7 / 10)$. As documented in our earlier study [11], the severe pain was explicitly addressed by the oncology team with an active pain management plan in a relatively high proportion of patient visits (83\% of all visits, including $97 \%$ of visits where the pain was deemed cancer- or treatment-related, and $57 \%$ of visits where the documented cause of pain was non-cancer related or unknown). In the majority of cases, these plans were carried out, and if they were not, it was usually due to patient preferences. However, an unexpected finding was the low number of visits that were scheduled within a short timeframe, which would have allowed reassessment of the pain scores.

In fact, the majority of patients' first follow-up visits were not captured in this study, as they fell outside the three-month window after the baseline visit was reviewed. Follow-up visit scheduling did not appear to be short-term, as 37 out of 96 patients received their next visit three months or later after baseline. At our centre, at the time of this study, follow-up visit timing was entirely at the discretion of the oncologist, and an electronic follow-up booking order, set with prepopulated, recommended follow-up timeframes, was not in use yet (as it is now). Therefore, the timing of the booked follow-up visit entirely reflects the conscious clinical decision of the oncologist. We therefore conclude that despite the initial attention given to the severe pain, in-clinic follow-up was often not arranged primarily to reassess pain levels in the short-term but was perhaps linked more to the expected progression of the underlying cancer.

The limiting factor in generalization of the results of this study is the relatively small number of patients actually seen in follow-up. It is unknown what occurred in many patients with respect to their pain management as a large proportion received no follow-up within three months after severe pain was documented on the ESAS. Nevertheless, for those patients for which follow-up data is available, pain documented by the ESAS decreased from baseline at each subsequent interval over the 12-week period. No associations were discovered between other ESAS scores and a change in pain level over 12 weeks. Similarly, Hwang et al. [13] described that no independent predictors of pain relief were identified over a three-week longitudinal study. These findings confirm the complex nature of pain and relate to difficulties faced in management across modalities. There was a correlation between length of time from baseline to last ESAS pain level. With increasing length of time from baseline, pain severity as rated by the ESAS decreased. The improvement in pain level as documented on the ESAS may be confounded by passage of time as opposed to a therapeutic response to the pain management plan.

Previous studies have shown similar findings in that pain ratings from admission to follow-up were decreased, and this reduction was not associated with treatment [14]. Deardoff et al. [14] proposed that at baseline, when patients are being evaluated for treatment, subjective pain level on a rating scale is inflated. When treatment was already initiated or completed and obtaining treatment was no longer a concern, patients may more accurately rate their pain. Paice [15] proposed that patients under-report their symptoms over time, as they do not want to burden their family or physician.

\section{Conclusions}

In this study of oncology follow-up after an initial patient presentation with severe pain, we found that only a minority of patients had oncology follow-up booked within three months. This leads to the conclusion that despite pain having been addressed at the initial visit, this assessment did not necessarily lead to follow-up in a timely manner to specifically reassess the pain. In a subset of the 96 cancer patients initially reporting severe pain, reduction in pain was observed over time, independent of several variables including type of pain and type of treatment. However, this finding was limited by small numbers available for follow-up. This study illustrates that pain level reassessment itself did not appear to be the main determinant of booking follow-up. Given the physical constraints of a typical cancer centre, perhaps 
alternative forms of support and follow-up, other than a clinic visit, could be considered when assessing a patient with severe pain. This could take the form of timed telephone calls, mail survey follow-up, or more precise communication to the primary healthcare providers with regard to pain management follow-up steps.

\section{Additional Information}

\section{Disclosures}

Human subjects: Consent was obtained by all participants in this study. Western University Health Sciences Research Ethics Board issued approval 106326. Animal subjects: All authors have confirmed that this study did not involve animal subjects or tissue. Conflicts of interest: In compliance with the ICMJE uniform disclosure form, all authors declare the following: Payment/services info: All authors have declared that no financial support was received from any organization for the submitted work. Financial relationships: All authors have declared that they have no financial relationships at present or within the previous three years with any organizations that might have an interest in the submitted work. Other relationships: All authors have declared that there are no other relationships or activities that could appear to have influenced the submitted work.

\section{Acknowledgements}

We would like to thank Larry Stitt and Frances Whiston for their valuable assistance with database management and statistical analysis.

\section{References}

1. Wells N, McDowell RM, Hendricks P, et al.: Cancer pain management in ambulatory care: can we link assessment and action to outcomes?. Support Care Cancer. 2011, 19:1865-1871. 10.1007/s00520-010-1030-7

2. van den Beuken-van Everdingen MH, de Rijke JM, Kessels AG, et al.: High prevalence of pain in patients with cancer in a large population-based study in The Netherlands. Pain. 2007, 132:312-320. 10.1016/j.pain.2007.08.022

3. Deandrea S, Montanari M, Moja L, et al.: Prevalence of undertreatment in cancer pain. A review of published literature. Ann Oncol. 2008, 19:1985-1991. 10.1093/annonc/mdn419

4. Yennurajalingham S, Kang JH, Hui D, et al.: Clinical response to an outpatient palliative care consultation in patients with advanced cancer and cancer pain. J Pain Symptom Manage. 2012, 44:340-350. 10.1016/j.jpainsymman.2011.09.014

5. Cleeland CS, Gonin R, Hatfield AK, et al.: Pain and its treatment in outpatients with metastatic cancer. N Engl J Med. 1994, 330:592-596. 10.1056/NEJM199403033300902

6. Mitera G, Zeiadin N, Kirou-Mauro A, et al.: Retrospective assessment of cancer pain management in an outpatient palliative radiotherapy clinic using the Pain Management Index. J Pain Symptom Manage. 2010, 39:259-267. 10.1016/j.jpainsymman.2009.07.005

7. Chow E, Doyle M, Li K, et al.: Mild, moderate, or severe pain categorized by patients with cancer with bone metastasis. J Palliat Med. 2006, 9:850-854. 10.1089/jpm.2006.9.850

8. Strohbuecker B, Mayer H, Evers GC, et al.: Pain prevalence in hospitalized patients in a German university teaching hospital. J Pain Symptom Manage. 2005, 29:498-506. 10.1016/j.jpainsymman.2004.08.012

9. Wells N, Hepworth JT, Murphy BA, et al.: Improving cancer pain management through patient and family education. J Pain Symptom Manage. 2003, 25:344-356. 10.1016/S08853924(02)00685-1

10. Bruera E, Kuehn N, Miller MJ, et al.: The Edmonton Symptom Assessment System (ESAS): a simple method for the assessment of palliative care patients. J Palliat Care. 1991, 7:6-9.

11. Sanatani MS, Kattan M, Moulin DE: Initial pain management plans in response to severe pain indicators on oncology clinic previsit questionnaires. Pain Res Manag. 2014, 19:309-312.

12. Farrar JT, Young JP Jr, LaMoreaux L, et al.: Clinical importance of changes in chronic pain intensity measured on an 11-point numerical pain rating scale. Pain. 2001, 94:149-158. 


\section{Cureus}

13. Hwang S, Chang V, and Kasimis B: Dynamic cancer pain management outcomes: the relationship between pain severity, pain relief, functional interference, satisfaction and global quality of life over time. J Pain Symptom Manage. 2002, 23:190-200.

14. Deardorff W, Rubin H, Scott D: Comprehensive multidisciplinary treatment of chronic pain: a follow-up study of treated and non-treated groups. Pain. 1991, 45:35-43. 10.1016/03043959(91)90162-Q

15. Paice J: Assessment of symptom clusters in people with cancer pain . J Natl Cancer Inst Monogr. 2004, 32:98-102. 10.1093/jncimonographs/lgh009 